\title{
Clinical, MRI findings and arthroscopic correlation of the meniscal injuries of the knee
}

\author{
Vijay Chandru ${ }^{1, *}$, Nagakiran K V², Anupama Chandrappa ${ }^{3}$, Ishani Patel ${ }^{4}$ \\ ${ }^{\mathbf{1}}$ Associate Professor, ${ }^{\mathbf{2}}$ Assistant Professor, ${ }^{3,4}$ Senior Resident, Dept. of Orthopaedics, ${ }^{1,3}$ J.S.S Medical College, JSS AHER, \\ Mysure, Karnataka, ${ }^{2}$ P.E.S. Institute of Medical Sciences and Research, Andhra Pradesh, ${ }^{4}$ V.S Hospital \& NHL Medical College, \\ Ahmedabad, Gujarat, India \\ *Corresponding Author: Vijay Chandru \\ Email: drvijay195@gmail.com
}

\begin{abstract}
The knee joint being very commonly injured secondary to road traffic accidents, contact sports activities and repetitive activities, in such cases especially in acute conditions its difficult to rely to arrive at a accurate clinical diagnosis depending only on the clinical tests to diagnose meniscal injuries because of the painful knee so the patient is subjected to many noninvasive imaging modalities like x-ray sonography and magnetic resonance imaging and later invasive procedures like diagnostic arthroscopy to detect the proper diagnosis. So we conducted a prospective in JSS hospital study to compare all the three modalities like clinical examination, MR imaging and diagnostic arthroscopy to know the meniscal injuries of the knee joint. There were 30 patients with 26 male and 4 female patients age ranging from 18 to 55 years. History of fall with twisting injury was most common. The sensitivity and specificity of clinical examination and MR imaging with respect to arthroscopy was $83.3 \%$ and $77.78 \%$ and $91.67 \%$ and $55.56 \%$ respectively. So diagnosing meniscal injuries is of great significance to decrease the morbidity of the patient.
\end{abstract}

Keywords: Clinical examination, MRI, Arthroscopy, Meniscal injuries.

\section{Introduction}

The knee joint being very commonly injured secondary to road traffic accidents, contact sports activities and repetitive activities, in such cases especially in acute and also in subacute condition conditions its difficult to rely to arrive at a accurate clinical diagnosis depending only on the clinical tests to diagnose meniscal injuries because of the painful knee so the patient is subjected to many noninvasive imaging modalities like x-ray sonography and Magnetic resonance imaging and later invasive procedures like diagnostic arthroscopy to detect the proper diagnosis. Magnetic resonance imaging has a better soft tissue delineation which is routinely done to the complex anatomy of the knee joint prior to diagnostic arthroscopy which can be diagnostic and therapeutic tool. ${ }^{1-3}$

Magnetic resonance imaging is important in preoperative planning to address the meniscal injuries and to whether it is amenable to repair or meniscectomy the sensitivity and specificity to detect medial and lateral meniscal injuries is $86-96 \%, 84-94 \%$ and 68 $86 \%$, 92-98\% respectively. Apart from these other meniscal lesions like parameniscal cyst can also be diagnosed.

\section{Aims and Objectives}

This study is aimed at comparing and correlating the clinical examination findings and MRI findings with arthroscopic findings to arrive at diagnosis of various meniscal injuries of the knee joint.

\section{Materials and Methods}

This study comprised 30 patients who were admitted in our hospital later subjected MRI after thorough clinical examination where some patients had MRI done elsewhere were also included in the study followed by diagnostic arthroscopy after taking the consent from the patients.

Study Design: Comparative study.

Place of Study: JSS Hospital department of orthopedics. Study Population: Patients with history of injury subjected to arthroscopy after thorough clinical examination and MRI.

Period of Study: September 2014 to May 2016.

Inclusion Criteria:

1. Pain in the knee secondary to twisting injury.

2. Locking of the knee joint present for more than 4 weeks.

3. Age of the patients between 18 to 55 .

4. Chronic knee pain with instability symptoms.

Exclusion Criteria

1. Patients already underwent meniscetomy ACL and PCL reconstruction.

2. Patients underwent MCL and LCL repair or reconstruction.

3. Patients symptoms suggestive of tumors of the knee joint structures.

4. Patient underwent surgeries like aneurysmal clips pacemakers etc.

5. Patients directly undergoing arthroscopy without prior MRI done.

\section{Mechanism of Injury}

Meniscal injuries occur secondary to trauma produced by the compressive load with a flexed knee 
and where as the type and location of the meniscal tear depends on the direction and magnitude of the force acting on the knee. Twisting injuries of the and sudden fall at home will also lead to tear in a degenerative knee joint.

\section{Classification}

Classified on the basis of etiology, site of the tear and radiological type of the tear and arthroscopic anatomical type of the tear like longitudinal, transverse, oblique, flap, parrot beak tear combination of tears and tears associated with meniscal cyst and discoid meniscus.

\section{Clinical Examination}

Palpatory findings like clicking, medial joint line or lateral joint line tenderness, Apleys grinding test, Mc Murray test, Childress test etc (Fig. 1-3).

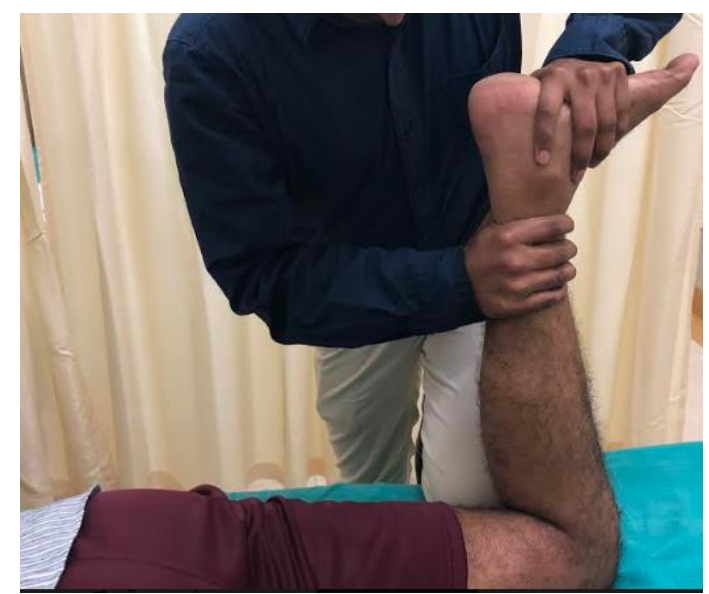

Fig. 1: Eliciting apleys grinding test for the meniscal injuries

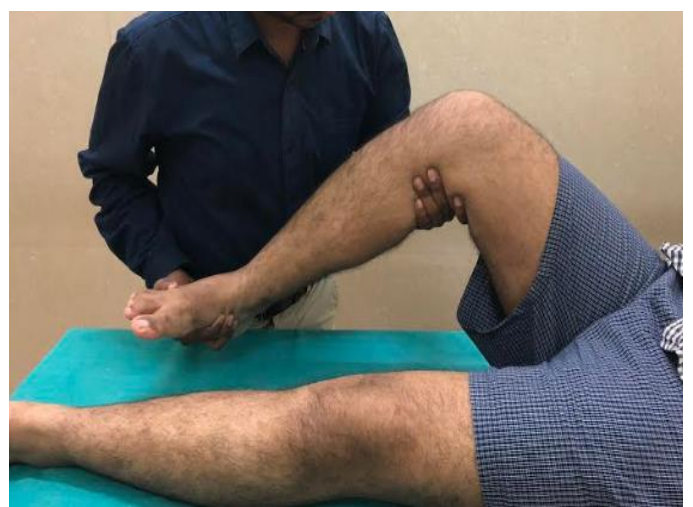

Fig. 2: Eliciting Mc Murrays test for the meniscal injury

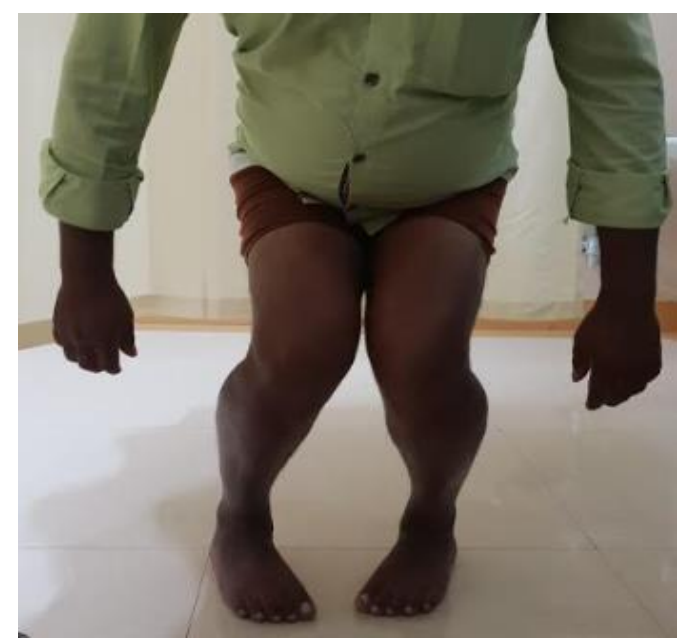

Fig. 3: Eliciting childress test for the meniscal injury

\section{Results}

There were 30 patients with 26 male and 4 female patients age ranging from 18 to 55 years. History of fall with twisting injury was most common and the right knee being more commonly injured. The sensitivity and specificity of physical and clinical examination for the lateral meniscal injuries with respect to arthroscopy is $75 \%$ and $77.27 \%$ respectively. The sensitivity and specificity for the lateral meniscal injuries of the MRI findings with respect to arthroscopy is $62.5 \%$ and $72.73 \%$ respectively. The sensitivity and specificity of the physical and Clinical examination findings of the medial meniscal injuries when compared to arthroscopy is $83.3 \%$ and $77.78 \%$ respectively. The sensitivity and specificity of the medial meniscal injuries of the MRI findings when compared to arthroscopy is $91.67 \%$ and $55.56 \%$ respectively. (Table 1,2 )

Table 1: Results for clinical examination in diagnosing medial and lateral meniscal injuries with respect to arthroscopy

\begin{tabular}{|l|c|c|}
\hline & Medial meniscus & Lateral meniscus \\
\hline Sensitivity & $83.3 \%$ & $75 \%$ \\
\hline Specificity & $77.78 \%$ & $77.27 \%$ \\
\hline
\end{tabular}

Table 2: Results for magnetic resonance imaging in diagnosing medial and lateral meniscal injuries with respect to arthroscopy

\begin{tabular}{|l|c|c|}
\hline & Medial meniscus & Lateral meniscus \\
\hline Sensitivity & $91.67 \%$ & $62.5 \%$ \\
\hline Specificity & $55.56 \%$ & $72.73 \%$ \\
\hline
\end{tabular}




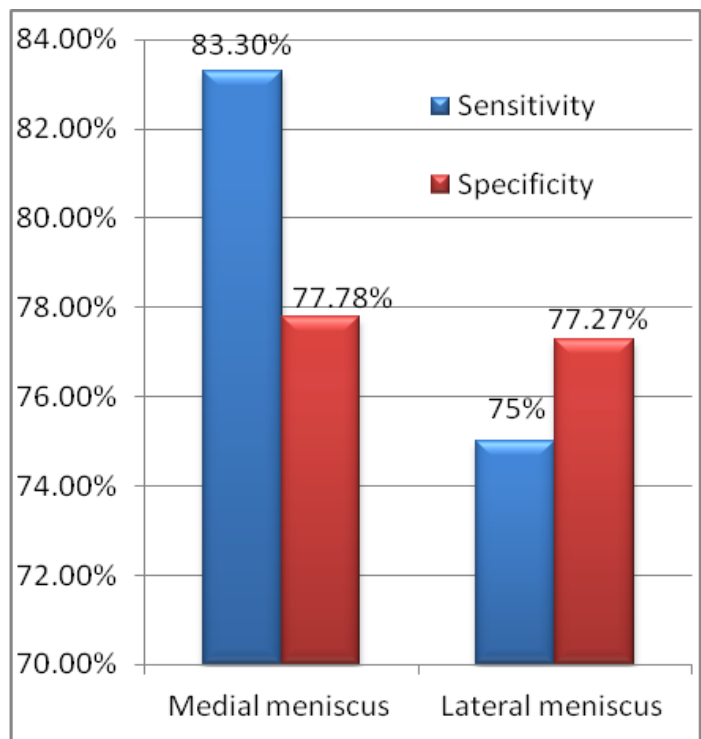

Fig. 4: Diagnostic accuracy of clinical examination versus arthroscopic evaluation in meniscal injuries



Fig. 5: Diagnostic accuracy of MRI versus arthroscopic evaluation in meniscal injuries

\section{Discussion}

This study is basically conducted to compare the how reliable the physical and clinical examination finding and the MRI findings of the meniscal injuries with the diagnostic findings of the arthroscopy. After a thorough clinical examination and noting down the type and site of the meniscal tears on MRI (Fig. 6), patient is subjected to diagnostic arthroscopy (Fig. 7) followed by therapeutic procedures if required totally there were 30 patients with 26 male and 4 female patients age ranging from 18 to 55 years. Males were commonly injured as study conducted by Fritz et al showed males are commonly injured as they are more involved in sports activities.

Medial meniscus was more commonly injured than lateral we found only 12 cases being diagnosed by arthroscopy when compared 14 cases which were clinically diagnosed so the sensitivity and specificity of the medial meniscal injury of the clinical examination findings will be is $83.33 \%$ and $77.78 \%$ when compared to diagnostic arthroscopic findings where as the MRI detected 19 cases so the sensitivity and specificity of MRI findings will be $91.67 \%$ and $55.56 \%$ when compared to diagnostic arthroscopic findings. Mohan et al study also concludes $88 \%$ accuracy of the clinical examination findings in diagnosing medial meniscal injuries where as Pappenport et al showed an accuracy of $90 \%$ in detecting meniscal injuries on MRI. Elvenes et al found sensitivity and specificity of $100 \%$ and $77 \%$ respectively where as positive and negative predictive value of MRI findings for medial meniscus tears was $71 \%$ and $100 \%$ in our study we have Sensitivity and specificity of $91.56 \%$ and $55.56 \%$ where as positive and negative predictive value is $57.89 \%$ and $90.91 \%$. In our present study we found that sensitivity, specificity, positive and negative predictive value of physical and clinical examination compared to diagnostic arthroscopy arthroscopy findings was less compared to the other studies. In our study we found that sensitivity, specificity, positive and negative predictive value of MRI compared to arthroscopy was less compared to the other studies.

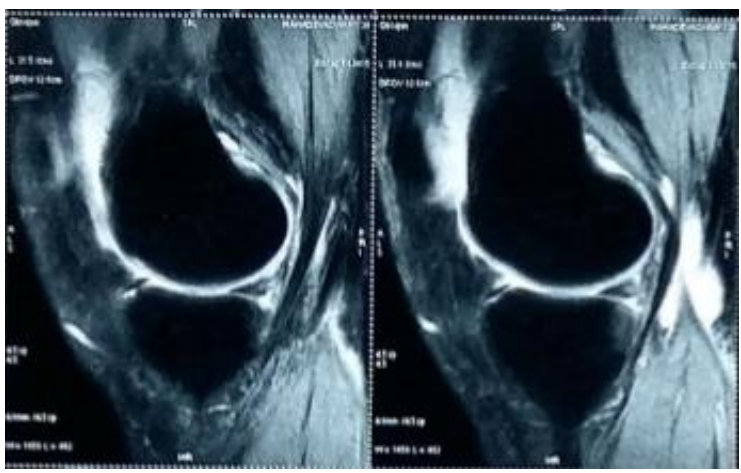

Fig. 6: T1 weighted image showing the posterior horn medial meniscal injury

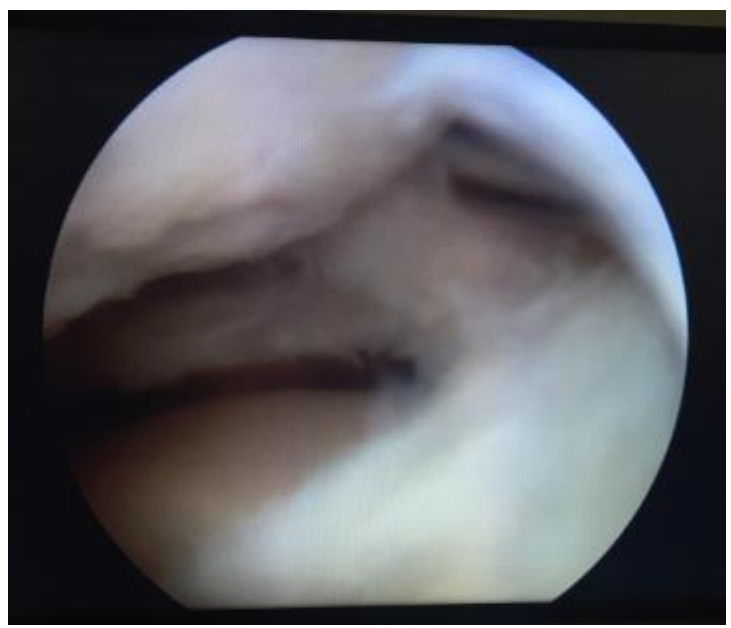

Fig. 7: Arthroscopic view of the posterior horn medial meniscal injury 
Diagnostic arthroscopy confirmed only 8 cases for lateral meniscal injuries out of the 11 cases which was diagnosed clinically and again arthroscopy confirmed 8 cases out of the 11 cases which was detected as per the MRI findings so sensitivity and specificity of clinical examination is $75 \%$ and $77.27 \%$ with respect to arthroscopy and sensitivity and specificity of the MRI is $62.5 \%$ and $72.73 \%$ with respect arthroscopy for the lateral meniscal injuries. Mohan et al in their study found $92 \%$ accuracy of the clinical examination findings for the lateral meniscal injury so if the MRI is used only in preoperative screening conditions this can be avoided provided the patients agree for diagnostic arthroscopy and proceed as they can save the expense of the MRI. As described by Mink et al there are misinterpretation of the meniscofemoral ligaments injury osteochondral flap avulsion which can mimic meniscal injuries and sometime as described by Crues et al MR imaging can separate the cases which are surgically significant from nonsignificant meniscal injuries may be useful in the noninvasive preoperative screening of suspected meniscal injuries of the knee joint. ${ }^{4,5}$

Rose et al also said MRI not necessary in the view of high cost in diagnosing menisal injuries if clinically found suspicion of meniscal tears. Gillies et al concluded sometimes negative findings by arthroscopy and MRI may be necessary to occasionally to remove a meniscus if the symptoms of knee pain persisting

Simonsen et al says arthroscopy should be conducted if there clinical evaluation of traumatic hemarthrosis present Boden et al supports that treatment plan will not change if the MRI is done after suspecting meniscal injuries clinically.

Overall accuracy of arthroscopy varies between $70 \%$ to $100 \%$ which depends on the skill and experience. This again keeps us in doubt regarding the MRI with arthroscopy findings. ${ }^{6-9}$

\section{Conclusion}

Its very important to diagnose and treat meniscal injuries if not diagnosed properly it will lead to morbidity with chronic pain later leading to degenerative joint disease by after eroding the articular cartilage. Clinical examination plays a crucial role in diagnosing meniscal injuries, MRI is an aid in radiological diagnosis especially in an acute condition but arthroscopy is the gold standard in arriving at the diagnosis and addressing the meniscal injuries at the same time. Where as in a clinically proven case of meniscal injury directly diagnostic arthroscopy can be done and will be able the expensive radiological investigative tool like MRI. Surgeons skill and experience will carry great value in diagnosing and treating such injuries since the demands of the patient is increasing especially in the persons involved in sports activities in such patients it's better to get the MRI done prior to explain to the patient in detail. Ultimately surgeon has to depend on his good clinical acumen and then correlating his findings with MRI and proceeding for the diagnostic arthroscopy and therapeutic procedures for the meniscal injuries

\section{Conflict of Interest: None}

\section{References}

1. Kaplan PA, Walker CW, Kilcoyne RF, Brown DE, Tusek D, Dussault RG. Occult fractures patterns of the knee associated with ACL tears. Assessment with MR imaging. Radiol. 1992;183:835-838.

2. Gray SD, Kalpan PA, Dussalt RG. Imaging of Knee: current status. OCNA. 1997;28(4):643-658.

3. Kean DM, Worthington BS, Preston BJ. Nuclear MRI of knee: examples of normal anatomy and pathology. $\mathrm{Br} \mathrm{J}$ Radiol. 1983;56:355-361.

4. Elvenes J, Jerome CP, Reikeras O, Johansen O. MRI as a screening procedure to avoid Arthroscopy for meniscal tears. Arch Orthop Trauma Surg. 2000;120(1- 2):14-16.

5. Crues JV 3rd, Mink J, Levy TL, Lotysch M, Stoller DW. Meniscal tears of the knee: accuracy of MR imaging. Radiol. 1987;164(2):445-448.

6. Rose NE, Gold SM. A comparison of accuracy between clinical examination and magnetic resonance imaging in the diagnosis of meniscal and anterior cruciate ligament tears. Arthroscopy. 1996;12:398-405.

7. Gillies, Seligson. Precision in the diagnosis of meniscal lesions: a comparison of clinical evaluation, arthrography, and arthroscopy. JBJS Am. 1979;61:343-346.

8. Simonsen, Jensen, Mouritsen, Lauritzen J. The accuracy of clinical examination of injury of the knee joint. Injury. 1984;16:96-101.

9. Boden, Labropoulos, Vailas. MR scanning of the acutely injured knee: sensitive, but is it cost effective? Arthroscopy. 1990;6:306-310.

How to cite this article: Chandru V, Nagakiran K
V, Chandrappa A, Patel I. Clinical, MRI findings
and arthroscopic correlation of the meniscal
injuries of the knee. Indian J Orthop Surg.
2018;4(4):390-393.

